S2K-Leitlinien „Einsatz von Exoskeletten im beruflichen Kontext zur Primär-, Sekundär- und Tertiärprävention von arbeitsassoziierten muskuloskelettalen Beschwerden“ - Leitlinienreport

\title{
S2K-Leitlinie
}

„Einsatz von Exoskeletten im beruflichen Kontext zur Primär-, Sekundär- und Tertiärprävention von arbeitsassoziierten muskuloskelettalen Beschwerden“

\author{
AWMF-Reg. Nr. \\ 002-046 \\ Leitlinienkoordinatoren \\ Dr. Benjamin Steinhilber \\ Dr. Tessy Luger \\ Institut für Arbeitsmedizin, Sozialmedizin und Versorgungsforschung am \\ Universitätsklinikum Tübingen
}

Autoren der Leitlinie

Benjamin Steinhilber, Tessy Luger, Peter Schwenkreis, Stefan Middeldorf, Hartmut Bork, Bernhard Mann, Alexander von Glinski, Thomas A. Schildhauer, Stephan Weiler, Martin Schmauder, Kai Heinrich, Gabriele Winter, Gerhard Schnalke, Peter Frener, Ralf Schick, Sascha Wischniewski, Matthias Jäger

Schlüsselwörter: Assistenzsystem, Prävention, Muskel-Skelett-Beschwerden, MuskelSkelett-Erkrankungen, Muskel-Skelett-Belastungen, Muskel-Skelett-Beanspruchungen, berufliche Belastungen

Keywords: Assistive device, prevention, musculoskeletal complaints, musculoskeletal disorders, musculoskeletal load, musculoskeletal strain, workload

\section{Geltungsbereich und Zweck}

○ Begründung für die Auswahl des Leitlinienthemas (s. DELBI-Kriterium 1)

Die Prävention arbeitsassoziierter Muskel-Skelett-Beschwerden (MSB) und Muskel-SkelettErkrankungen (MSE) hat vor dem Hintergrund der Prävalenz von MSB und MSE und damit verbundenen hohen Belastung der Gesundheitssysteme, Wirtschaft und der Betroffenen selbst einen hohen Stellenwert. Ob die Verwendung von Exoskeletten zur Prävention von MSB oder gar MSE beiträgt, wird aktuell kontrovers diskutiert.

$\circ$ Zielorientierung der Leitlinie (s. DELBI-Kriterium 1)

Die vorliegende Leitlinie thematisiert Einsatzmöglichkeiten von Exoskeletten in der betrieblichen Anwendung zur Prävention von Muskel-Skelett-Beschwerden (MSB). Dabei werden die Bereiche Primär-, Sekundär-, und Tertiärprävention von MSB differenziert sowie auch allgemeine Empfehlungen zur Nutzung, Implementierung von Exoskeletten und zur Gefährdungsbeurteilung gegeben.

○ Patientenzielgruppe (s. DELBI-Kriterium 3) 
S2K-Leitlinien „Einsatz von Exoskeletten im beruflichen Kontext zur Primär-, Sekundär- und Tertiärprävention von arbeitsassoziierten muskuloskelettalen Beschwerden“ - Leitlinienreport

Gesunde Beschäftigte, deren Muskel-Skelett-System vor Beschwerden und Erkrankungen geschützt werden soll (Primärprävention). Beschäftigte mit MSB oder MSE, die vor einer Verschlimmerung bzw. Manifestierung einer Erkrankung bewahrt werden sollen bzw. die eine Teilhabe an beruflichen Tätigkeiten wiedererlangen sollen.

$\circ$ Versorgungsbereich (s. DELBI-Kriterium 3)

Der Versorgungsbereich ist die arbeits- und betriebsmedizinische Beratung und Vorsorge sowie die ergonomische Arbeitsgestaltung zum Erhalt der Arbeitsfähigkeit, die Vermeidung von Manifestation und Verschlimmerung arbeitsbedingter Muskel-Skelett-Erkrankungen und -Beschwerden sowie die berufliche Wiedereingliederung.

$\circ$ Anwenderzielgruppe/Adressaten (s. DELBI-Kriterium 6)

Die Leitlinie richtet sich an Betriebsärztinnen und Betriebsärzte, Arbeitsmedizinerinnen und Arbeitsmediziner, Ergonominnen und Ergonomen, Fachkräfte für Arbeitssicherheit, Arbeitgeberinnen und Arbeitgeber und dient zur Information für alle weiteren Akteurinnen und Akteure des praktischen Arbeitsschutzes.

\section{Zusammensetzung der Leitliniengruppe: Beteiligung von Interessensgruppen}

○ Repräsentativität der Leitliniengruppe: Beteiligte Berufsgruppen (s. DELBI-Kriterium 4) Die Mitglieder der Leitliniengruppe sind Ärztinnen und Ärzte sowie Wissenschaftlerinnen und Wissenschaftler aus den Bereichen Arbeitsmedizin, Arbeitswissenschaft, Ergonomie, Arbeitsschutz, Biomechanik, Orthopädie, Orthopädische Chirurgie und Unfallchirurgie, Neurologie, Schmerzforschung sowie Sozialmedizin und Prävention. Zudem sind Vertreter der Deutschen Gesetzlichen Unfallversicherung, verschiedener Berufsgenossenschaften, der Bundesanstalt für Arbeitsschutz und Arbeitsmedizin und aus dem Bereich der Rehabilitation mit einem hohen Bezug zur beruflichen Praxis in der Leitliniengruppe vertreten.

Tabelle 1: MandatsträgerInnen und stellvertretende MandatsträgerInnen

\begin{tabular}{cc} 
Mandatsträger & Fachgesellschaft / Organ isation \\
\hline Dr. rer. nat. Benjamin Steinhilber & Deutsche Gesellschaft für Arbeitsmedizin und \\
Umweltmedizin e.V.
\end{tabular}


S2K-Leitlinien „Einsatz von Exoskeletten im beruflichen Kontext zur Primär-, Sekundär- und Tertiärprävention von arbeitsassoziierten muskuloskelettalen Beschwerden“ - Leitlinienreport

Tabelle 2 BeraterInnen

\begin{tabular}{|c|c|}
\hline $\begin{array}{c}\text { Weitere TeilnehmerInnen als } \\
\text { BeraterInnen }\end{array}$ & Funktion \& Fachgesellschaft/ Organisation \\
\hline Dr. Kai Heinrich & Berater, Deutsche Gesetzliche Unfallversicherung \\
\hline Dr.-Ing. Sascha Wischniewski & Berater, Bundesanstalt für Arbeitsschutz und Arbeitsmedizin \\
\hline Dr.-Ing. Gabriele Winter & $\begin{array}{c}\text { Beraterin, Berufsgenossenschaft Verkehrswirtschaft Post- } \\
\text { Logistik Telekommunikation }\end{array}$ \\
\hline Peter Frener & Berater, Berufsgenossenschaft Holz und Metall \\
\hline Ralf Schick & Berater, Berufsgenossenschaft Handel und Warenlogistik \\
\hline Gerhard Schnalke & $\begin{array}{l}\text { Berater, EFL-Akademie (Evaluation funktioneller } \\
\text { Leistungsfähigkeit nach Isernhagen) }\end{array}$ \\
\hline
\end{tabular}

O Repräsentativität der Leitliniengruppe: Beteiligung von Patienten (s. DELBI-Kriterium 5)

Arbeitgeberinnen und Arbeitgeber sowie Beschäftigte waren nicht direkt an der Leitlinienerstellung beteiligt, da diese Assistenzsysteme zum Zeitpunkt der Leitlinienerstellung noch kaum im Routineeinsatz waren. Dennoch wurden Firmen, in denen zum Zeitpunkt der Leitlinienerstellung, Exoskelette erprobt wurden, nach ihren Erfahrungen und Intentionen bei der Nutzung von Exoskeletten befragt.

\section{Methodologische Exaktheit}

\section{Recherche, Auswahl und Bewertung wissenschaftlicher Belege (Evidenzbasierung)}

$\circ$ Formulierung von Schlüsselfragen (s. DELBI-Kriterium 2)

Folgende Schlüsselfragen wurden für die einzelnen Kapitel der Leitlinie im Rahmen der Literaturrecherche bearbeitet:

\section{Allgemeine Empfehlungen}

- Wie können Exoskelette sinnvoll in die betriebliche Praxis eingeführt werden?

- Gibt es günstige bzw. ungünstige Umgebungsbedingungen für den Einsatz von Exoskeletten in der betrieblichen Praxis?

\section{Primärprävention}

- Kann durch den Einsatz von Exoskeletten die Belastung oder Beanspruchung der Muskulatur (der oberen Extremitäten [einschl. Schulter, Kopf/Hals/Nacken], des Rumpfes [einschl. der Wirbelsäule], der unteren Extremitäten [einschl. Hüfte/Becken]) verringert werden?

- Kann durch den Einsatz von Exoskeletten die körperliche Belastung (der oberen Extremitäten, des Rumpfes, der unteren Extremitäten) verringert werden?

- Kann durch den Einsatz von Exoskeletten eine subjektiv empfundene Belastungsminderung (in den oberen Extremitäten, im Rumpf, in den unteren Extremitäten) erreicht werden?

- Können durch den Einsatz von Exoskeletten Belastungen oder Beanspruchungen (der oberen Extremitäten, des Rumpfes, der unteren Extremitäten) erhöht werden bzw. zusätzliche Belastungs- oder Beanspruchungsarten entstehen?

Sekundär- und Tertiärprävention

- Kann durch Verwendung eines Exoskeletts eine Entlastung der geschädigten Körperstruktur erreicht werden? 
S2K-Leitlinien „Einsatz von Exoskeletten im beruflichen Kontext zur Primär-, Sekundär- und Tertiärprävention von arbeitsassoziierten muskuloskelettalen Beschwerden“ - Leitlinienreport

- Gibt es Kontraindikationen für den Einsatz eines Exoskeletts im Rahmen der Sekundär- und Tertiärprävention?

- Welche Einsatzdauer eines Exoskeletts ist für den Einsatz im Rahmen der Sekundär- und Tertiärprävention sinnvoll?

Ergänzend wurden folgende Fragen an 19 Firmen und neun Exoskelettherstellerinnen und hersteller gestellt (von sieben Firmen und einem Exoskeletthersteller wurden die Fragen beantwortet).

\section{Primärprävention}

- Stellen Sie Ihren Beschäftigten Exoskelette zur Prävention von Muskel-SkelettErkrankungen zur Verfügung?

\section{Sekundärprävention}

- Stellen Sie Ihren (leistungsgewandelten) Beschäftigten mit Erkrankungen des MuskelSkelett-Systems Exoskelette zur Verfügung, um ein Voranschreiten der Erkrankung zu verhindern?

\section{Tertiärprävention}

- Stellen Sie Ihren (leistungsgewandelten) Beschäftigten nach krankheitsbedingter Arbeitsunfähigkeit Exoskelette zur beruflichen Wiedereingliederung zur Verfügung?

\section{Fragen unabhängig, in welchem Präventionsbereich das Exoskelett eingesetzt wird}

- Wie werden Exoskelette eingeführt? Gibt es Schulungsmaßnahmen?

- Für welche Tätigkeiten werden Exoskelette genutzt?

- Unter welchen Umweltbedingungen werden Exoskelette genutzt (z.B. nicht bei Kälte oder Hitze)?

- In welchem Umfang je Person werden Exoskelette eingesetzt?

A) Bitte geben Sie die durchschnittliche ununterbrochene Tragedauer an.

B) Bitte geben Sie die Häufigkeit der Nutzung pro Tag, Woche, Monat an.

- Ist die Nutzung des Exoskeletts für die Beschäftigten freiwillig?

\section{○ Verwendung existierender Leitlinien zum Thema (s. DELBI-Kriterien 30-34)}

Es gibt bislang keine Leitlinie, die den Einsatz von Exoskeletten zur Unterstützung von Funktionen des Skelett- und Muskelsystems adressiert.

\section{○ Systematische Literaturrecherche (s. DELBI-Kriterium 8)}

Das in Abbildung 1 dargestellte Flussdiagramm zeigt, in welchen Datenbanken gesucht wurde. Die Suchstrategie wurde im ersten Leitlinientreffen entwickelt. Dabei einigte sich die Leitliniengruppe darauf eine Recherche mit möglichst wenigen Einschränkungen durchzuführen, d.h. weder zum Studientyp, dem Setting oder den eingeschlossenen Probanden. Es wurde ausschließlich deutsch- und englischsprachige Literatur einbezogen. Die gefundene Literatur wurde gemäß den Kapiteln der Leitlinie kategorisiert und schließlich von mehreren Arbeitsgruppen zur Ableitung von Empfehlungen genutzt. Zusätzlich wurde sogenannte graue Literatur aus eigenen Quellen der Leitlinienautoren z.B. von wissenschaftlichen Kongressen eingeschlossen. Obwohl, wie im vorherigen Unterkapitel bereits beschrieben, keine systematische Bewertung der methodischen Qualität erfolgte, wurden die wesentlichen Daten der gesichteten Literatur extrahiert und dabei auch Aspekte der methodischen Qualität wie z.B. die untersuchte Fallzahl der Studien berücksichtigt. Die Recherche wurde am 23. und 25. Januar 2019 durchgeführt. 
S2K-Leitlinien „Einsatz von Exoskeletten im beruflichen Kontext zur Primär-, Sekundär- und Tertiärprävention von arbeitsassoziierten muskuloskelettalen Beschwerden“ - Leitlinienreport
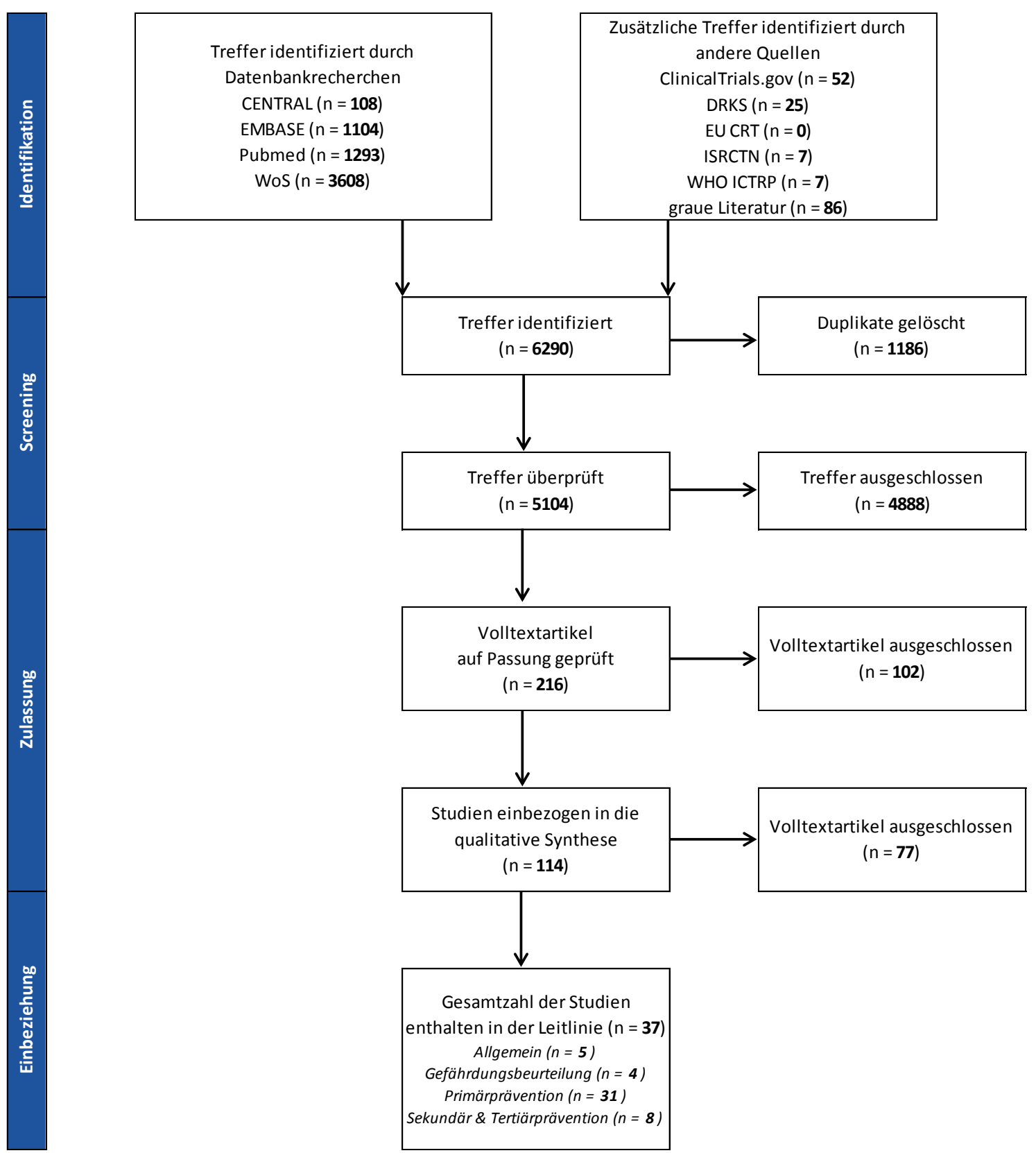

Abbildung 1 Flussdiagramm - Literatursuche. WoS Web of Science, DRKS Deutsches Register Klinische Studien; EU CRT European Union Clinical Trials Register; ISRCNT International Standard Randomised Controlled Trial Number; WHO ICTRP World Health Organization Clinical Trial Registry Platform, graue Literatur aus eigenen Quellen der Leitlinienautoren (z. B. aus Kongressbänden)

\section{$\circ$ Auswahl der Evidenz (s. DELBI-Kriterium 9)}

Aus den gesichteten Quellen wurden die wesentlichen Ergebnisse extrahiert und zusammengestellt. Auf dieser Basis wurde der Leitlinientext geschrieben und Empfehlungen sowie Kernaussagen entwickelt.

○ Bewertung der Evidenz (mit Hilfe strukturierter Checklisten, z.B. SIGN)

Wurde in der vorliegenden S2k-Leitlinie nicht durchgeführt.

\section{o Erstellung von Evidenztabellen}

Wurde in der vorliegenden S2k-Leitlinie nicht durchgeführt. 
S2K-Leitlinien „Einsatz von Exoskeletten im beruflichen Kontext zur Primär-, Sekundär- und Tertiärprävention von arbeitsassoziierten muskuloskelettalen Beschwerden“ - Leitlinienreport

\section{Formulierung der Empfehlungen und strukturierte Konsensfindung}

\section{○ Formale Konsensfindung: Verfahren und Durchführung (s. DELBI-Kriterium 10)}

Die strukturierte Konsensfindung erfolgte im Rahmen eines nominalen Gruppenprozesses während der Konsensuskonferenz am 1. Oktober 2019 unter unabhängiger Moderation durch eine Vertreterin der AWMF (Dr. Susanne Blödt). Im Vorfeld der Konsensuskonferenz erhielten alle Mitglieder der Leitliniengruppe eine Einladung zur Teilnahme sowie eine Übersicht der zur Abstimmung stehenden Kernaussagen und Empfehlungen. Die strukturierte Konsensfindung erfolgte nach dem nominalen Gruppenprozess wie folgt: Vorstellung der Kernaussagen und Empfehlungen, inhaltliche Rückfragen, Abstimmung jeder Empfehlung und ggf. Alternativvorschlägen, bei Nichterreichen eines Konsens Diskussion und erneute Abstimmung, ggf. Wiederholung bis zum Erreichen eines Konsens (> 75\%) bzw. starken Konsens (> 95\%). Die Prüfung des Konsensusgrades der jeweiligen Kernaussage/Empfehlung erfolgte jedoch nur von den Mandatsträgern der Fachgesellschaften. Allen anderen Teilnehmern und Teilnehmerinnen der Leitliniengruppe war lediglich die Diskussion und das Unterbreiten von Alternativvorschlägen zu den Kernaussagen und Empfehlungen möglich.

Nach der Konsensuskonferenz wurden eine Exoskelett-Definition und wenige Empfehlungen, im Rahmen einer DELPHI Abstimmung konsentiert. Die DELPHI-Abstimmungen wurden per Email und mit dem Abstimmungsformular auf der Internetseite der AWMF (https://www.awmf.org/leitlinien/awmf-regelwerk/Il-entwicklung.html; Zugriff 03.03.2020) durchgeführt. Vorschläge zu etwaigen Änderungen zu den Kernaussagen/Empfehlungen der DELPHI-Abstimmung konnten von jedem Mitglied der Leitliniengruppe unterbreitet werden. Die Abstimmung selbst wurde wiederum nur von den Mandatsträgern der Fachgesellschaften durchgeführt. Die nach der ersten Runde eingegangenen Änderungsvorschläge wurden in den Leitlinientext eingearbeitet und wiederum an die Leitliniengruppe versendet. Alle Empfehlungen und die Definition wurden in der 2. Runde mit starkem Konsens verabschiedet.

○ Berücksichtigung von Nutzen, Nebenwirkungen, relevanten Outcomes (s. DELBI-Kriterium 11)

Aufgrund der Neuheit von Exoskeletten zur Unterstützung beruflicher Tätigkeiten und der fehlenden wissenschaftlichen Erkenntnisse im Hinblick auf eine mögliche Prävention von Muskel-Skelett-Beschwerden und -Erkrankungen durch Exoskelette wurden insbesondere auch Aspekte der möglichen Nebenwirkungen durch Exoskelette beachtet. Hier fehlt jedoch ebenso die wissenschaftliche Evidenz wie für die präventive Wirkung auf das Muskel-SkelettSystem. Daher thematisiert die Leitlinie in den Empfehlungen $11-20$ einerseits die Freiwilligkeit der Exoskelettnutzung, mögliche Kontraindikationen für eine Anwendung sowie eine engmaschige Beobachtung / Betreuung durch die betriebsbetreuende Ärztin bzw. den betriebsbetreuenden Arzt.

\section{O Formulierung der Empfehlungen und Vergabe von Evidenzgraden und/ oder}

Empfehlungsgraden (s. DELBI-Kriterium 12)

Die Empfehlungen dieser Leitlinie wurden von Arbeitsgruppen (AG) entsprechend den Kapiteln dieser Leitlinie (AG Primärprävention, AG Sekundär-/Tertiärprävention, AG Allgemeine Empfehlungen, AG Gefährdungsbeurteilung) erarbeitet. Die AGs verwendeten dafür die Quellen der standardisierte Literaturrecherche, ihr Expertenwissen sowie die Angaben von sieben Firmen und einem Exoskeletthersteller zur Nutzung von Exoskeletten, die auf einen Fragebogen geantwortet haben.

Jede AG hat die Literatur nach einem einheitlichen Vorgehen gesichtet und die wesentlichen Informationen extrahiert, so dass auch methodische Aspekte zum Studiendesign und der untersuchten Population berücksichtigt wurden. Die Empfehlungsstärke wurde sprachlich durch soll für eine starke, sollte und kann für eine schwache Empfehlung ausgedrückt und wurde im Rahmen des Konsensusverfahrens festgelegt. 
S2K-Leitlinien „Einsatz von Exoskeletten im beruflichen Kontext zur Primär-, Sekundär- und Tertiärprävention von arbeitsassoziierten muskuloskelettalen Beschwerden“ - Leitlinienreport

\section{Externe Begutachtung und Verabschiedung}

$\circ$ Pilottestung (s. DELBI-Kriterium 7)

Eine Pilottestung wurde nicht durchgeführt.

o Externe Begutachtung (s. DELBI-Kriterium 13)

Diese Leitlinie stand vom 1. Juni bis zum 3. Juli 2020 als Konsultationsfassung auf der AWMF Webseite. Während dieses Zeitraums konnte die Leitlinie kommentiert werden. Es gab nur redaktionelle Kommentare, die entsprechend umgesetzt wurde.

\section{- Verabschiedung durch die Vorstände der herausgebenden Fachgesellschaften/Organisationen}

Die Leitlinie wurde am 16. April 2020 an die Vorstände der beteiligten Fachgesellschaften zur Freigabe übergeben. Die Leitlinie wurde von den beteiligten Fachgesellschaften freigegeben, wobei Rückmeldung und Anmerkungen der Vorstände innerhalb der Autorengruppe dieser Leitlinie besprochen und entsprechende Ergänzungen im Leitlinientext vorgenommen wurden.

\section{Redaktionelle Unabhängigkeit}

\section{o Finanzierung der Leitlinie (s. DELBI-Kriterium 22)}

- Die Arbeit der Leitliniengruppe erfolgte ehrenamtlich bzw. auf Kosten der beteiligten Fachgesellschaft/Institution oder des jeweiligen Arbeitgebers.

- Die finanziellen Mittel für die Moderation des ersten Leitlinientreffens und der Konsensuskonferenz (beim 1. Leitlinientreffen nur Reisekosten) sowie die Personalmittel für die Leitlinienkoordination wurden vom Institut für Arbeitsmedizin, Sozialmedizin und Versorgungsforschung am Universitätsklinikum Tübingen bereitgestellt.

- Die Räume und die Verpflegung beim 1. Leitlinientreffen (Auftaktsitzung) wurden vom Institut für Arbeitsmedizin, Sozialmedizin und Versorgungsforschung am Universitätsklinikum Tübingen bereitgestellt. Beim 2. Leitlinientreffen (Arbeitstreffen) wurden Räume und Verpflegung von der Berufsgenossenschaft Handel und Warenlogistik bereitgestellt, beim 3. Leitlinientreffen (Arbeitstreffen) von der Berufsgenossenschaft Holz und Metall. Beim 4. Treffen (Konsensuskonferenz) wurden Räume und Verpflegung vom BG Universitätsklinikum Bergmannsheil Bochum zur Verfügung gestellt.

\section{○ Darlegung von und Umgang mit potenziellen Interessenkonflikten (s. DELBI-Kriterium 23)}

Alle an der Erstellung dieser Leitlinie beteiligten Personen haben ihre Interessen schriftlich mit Hilfe des von der AWMF bereitgestellten Formblattes (Stand 17.01.2018) erklärt. Diese Erklärungen wurden von den Leitlinienkoordinatoren zusammengefasst und können im Leitlinienreport eingesehen werden. Die Bewertung dieser Erklärungen und ob Interessenskonflikte vorliegen wurde innerhalb der Leitliniengruppe inklusive einer Vertreterin (Dr. Susanne Blödt) der AWMF vor der Konsensusfindung durchgeführt. Die Interessenskonflikterklärungen aller stimmberechtigten Mandatsträger wurden gemeinsam gesichtet und als gering [z.B. exoskelettbezogene Forschung], moderat [z.B. vergütete Beratung von Exoskeletthersteller-Firmen] oder hoch [z.B. finanzielle Anteile am Verkauf von Exoskeletten] eingestuft. Ein moderater oder hoher Interessenskonflikt würde den Ausschluss von der Abstimmung der Leitlinienempfehlungen bedeuten. Zwar wurde zweimal ein moderater Interessenskonflikt festgestellt, allerdings waren diese beiden Mandatsträger zur Abstimmung der Empfehlungen nicht anwesend. Ferner wurde von der Leitliniengruppe beschlossen, dass alle Interessenskonflikte, auch die der Personen in beratender Funktion, offengelegt werden, um hier ein hohes Maß an Transparenz zu gewährleisten. Als protektive Faktoren die einer Verzerrung durch Interessenkonflikte entgegen wirken kann die pluralistische Leitliniengruppe, die strukturierte Konsensfindung unter neutraler Moderation, 
S2K-Leitlinien „Einsatz von Exoskeletten im beruflichen Kontext zur Primär-, Sekundär- und Tertiärprävention von arbeitsassoziierten muskuloskelettalen Beschwerden“ - Leitlinienreport

die Diskussion der Interessenserklärung zu Beginn der Konsensuskonferenz und die öffentliche Konsultation angesehen werden 
S2K-Leitlinien „Einsatz von Exoskeletten im beruflichen Kontext zur Primär-, Sekundär- und Tertiärprävention von arbeitsassoziierten muskuloskelettalen Beschwerden“ Leitlinienreport

Tabelle 3: Erklärung von Interessen und Umgang mit Interessenkonflikten

Im Folgenden sind die Interessenerklärungen als tabellarische Zusammenfassung dargestellt sowie die Ergebnisse der Interessenkonfliktbewertung und Maßnahmen, die nach Diskussion der Sachverhalte von der Leitliniengruppe beschlossen und im Rahmen der Konsensuskonferenz umgesetzt wurden.

\begin{tabular}{|c|c|c|c|c|c|c|c|}
\hline $\begin{array}{l}\text { Berater- } \\
\text { bzw. } \\
\text { Gutachter- } \\
\text { tätigkeit }\end{array}$ & $\begin{array}{l}\text { Mitarbeit in } \\
\text { einem } \\
\text { Wissenschaftlic } \\
\text { hen Beirat } \\
\text { (advisory } \\
\text { board) }\end{array}$ & $\begin{array}{l}\text { Bezahlte } \\
\text { Vortrags- } \\
\text { /oder } \\
\text { Schulungs- } \\
\text { tätigkeit }\end{array}$ & $\begin{array}{l}\text { Bezahlte } \\
\text { Autoren- } \\
\text { /oder } \\
\text { Coautoren- } \\
\text { schaft }\end{array}$ & $\begin{array}{l}\text { Forschungs } \\
\text {-vorhaben/ } \\
\text { Durchführu } \\
\text { ng } \\
\text { klinischer } \\
\text { Studien }\end{array}$ & $\begin{array}{l}\text { Eigentümer- } \\
\text { interessen } \\
\text { (Patent, } \\
\text { Urheberrecht } \\
\text { 'Aktienbesitz) }\end{array}$ & Indirekte Interessen & $\begin{array}{l}\text { Von COI betroffene } \\
\text { Themen der Leitlinie }{ }^{1} \text {, } \\
\text { (Einstufung bzgl. der } \\
\text { Relevanz), } \\
\text { Konsequenz }\end{array}$ \\
\hline
\end{tabular}

MandatsträgerInnen und stellvertretende MandatsträgerInnen

\begin{tabular}{|c|c|c|c|c|c|c|c|c|}
\hline $\begin{array}{l}\text { Dr. Benjamin } \\
\text { Steinhilber }\end{array}$ & $\begin{array}{l}\text { Ja (in einem } \\
\text { Forschungs } \\
\text { projekt mit } \\
\text { thematisch } \\
\text { en Bezug } \\
\text { zur } \\
\text { Leitlinie, } \\
\text { gefördert } \\
\text { durch das } \\
\text { Bundesmini } \\
\text { sterium für } \\
\text { Arbeit und } \\
\text { Soziales) }\end{array}$ & $\mathrm{Ja}$ & Ja & $\mathrm{Ja}$ & Ja & Nein & $\begin{array}{l}\text { Mitglied: Deutsche Gesellschaft für Arbeitsmedizin } \\
\text { und Umweltmedizin e. V. } \\
\text { Gesellschaft für Arbeitswissenschaft e. V. } \\
\text { International Society of Electromyography }\end{array}$ & $\begin{array}{l}\text { alle (gering), keine } \\
\text { Konsequenz }\end{array}$ \\
\hline Dr. Tessy Luger & Nein & Nein & $\mathrm{Ja}$ & $\mathrm{Ja}$ & Ja & Nein & $\begin{array}{l}\text { Mitglied: Deutsche Gesellschaft für Arbeitsmedizin } \\
\text { und Umweltmedizin e. V. }\end{array}$ & $\begin{array}{l}\text { alle (gering), keine } \\
\text { Konsequenz }\end{array}$ \\
\hline $\begin{array}{l}\text { Prof. Dr. med. } \\
\text { Peter } \\
\text { Schwenkreis }\end{array}$ & Nein & Nein & Nein & Nein & $\mathrm{Ja}$ & Nein & $\begin{array}{l}\text { Mitglied: Deutsche Gesellschaft für Neurologie e. V. } \\
\text { Deutsche Gesellschaft für Klinische } \\
\text { Neurophysiologie e. V. }\end{array}$ & $\begin{array}{l}\text { alle (gering), keine } \\
\text { Konsequenz }\end{array}$ \\
\hline
\end{tabular}


S2K-Leitlinien „Einsatz von Exoskeletten im beruflichen Kontext zur Primär-, Sekundär- und Tertiärprävention von arbeitsassoziierten muskuloskelettalen Beschwerden“ Leitlinienreport

\begin{tabular}{|c|c|c|c|c|c|c|c|c|}
\hline & $\begin{array}{l}\text { Berater- } \\
\text { bzw. } \\
\text { Gutachter- } \\
\text { tätigkeit }\end{array}$ & $\begin{array}{l}\text { Mitarbeit in } \\
\text { einem } \\
\text { Wissenschaftlic } \\
\text { hen Beirat } \\
\text { (advisory } \\
\text { board) }\end{array}$ & $\begin{array}{l}\text { Bezahlte } \\
\text { Vortrags- } \\
\text { /oder } \\
\text { Schulungs- } \\
\text { tätigkeit }\end{array}$ & $\begin{array}{l}\text { Bezahlte } \\
\text { Autoren- } \\
\text { /oder } \\
\text { Coautoren- } \\
\text { schaft }\end{array}$ & $\begin{array}{l}\text { Forschungs } \\
\text {-vorhaben/ } \\
\text { Durchführu } \\
\text { ng } \\
\text { klinischer } \\
\text { Studien }\end{array}$ & $\begin{array}{l}\text { Eigentümer- } \\
\text { interessen } \\
\text { (Patent, } \\
\text { Urheberrecht } \\
\text { ', } \\
\text { Aktienbesitz) }\end{array}$ & Indirekte Interessen & $\begin{array}{l}\text { Von COI betroffene } \\
\text { Themen der Leitlinie }{ }^{1} \text {, } \\
\text { (Einstufung bzgl. der } \\
\text { Relevanz), } \\
\text { Konsequenz }\end{array}$ \\
\hline & & & & & & & $\begin{array}{l}\text { Deutsche Schmerzgesellschaft e. V. } \\
\text { Deutsche Gesellschaft für Neurowissenschaftliche } \\
\text { Begutachtung e. V. } \\
\text { International Association fort he Study of Pain }\end{array}$ & \\
\hline $\begin{array}{l}\text { Dr. med. } \\
\text { Hartmut Bork }\end{array}$ & Nein & Nein & Nein & Nein & Nein & Nein & $\begin{array}{l}\text { Mitglied: Deutsche Gesellschaft für Orthopädie und } \\
\text { Orthopädische Chirurgie e. V. } \\
\text { Deutsche Gesellschaft für Orthopädie und } \\
\text { Unfallchirurgie e. V. }\end{array}$ & $\begin{array}{l}\text { alle (gering), keine } \\
\text { Konsequenz }\end{array}$ \\
\hline $\begin{array}{l}\text { PD. Dr. med. } \\
\text { Stephan Weiler }\end{array}$ & Nein & Nein & $\mathrm{Ja}$ & $\mathrm{Ja}$ & $\mathrm{Ja}$ & Nein & $\begin{array}{l}\text { Mitglied: Deutsche Gesellschaft für Arbeitsmedizin } \\
\text { und Umweltmedizin e. V. } \\
\text { Verband Deutscher Betriebs- und Werksärzte e.V. } \\
\text { Deutsche Gesellschaft für Sozialmedizin und } \\
\text { Prävention e. V. } \\
\text { Deutsches Netzwerk Evidenzbasierte Medizin } \\
\text { Arbeitsgrupp Exoskelette der Berufsgenossenschaft } \\
\text { Holz und Metall }\end{array}$ & $\begin{array}{l}\text { alle (gering), keine } \\
\text { Konsequenz }\end{array}$ \\
\hline $\begin{array}{l}\text { Dr. med. Stefan } \\
\text { Middeldorf }\end{array}$ & $\mathrm{Ja}$ & Nein & Nein & Nein & Nein & Nein & Mitglied: Deutsche Schmerzgesellschaft e. V. & $\begin{array}{l}\text { alle (moderat), } \\
\text { Stimmenthaltung }\end{array}$ \\
\hline $\begin{array}{l}\text { Prof. Dr.-Ing. } \\
\text { Martin }\end{array}$ & Nein & Nein & Nein & Nein & Nein & Nein & Mitglied: Gesellschaft für Arbeitswissenschaft e. V. & alle (gering), keine \\
\hline
\end{tabular}


S2K-Leitlinien „Einsatz von Exoskeletten im beruflichen Kontext zur Primär-, Sekundär- und Tertiärprävention von arbeitsassoziierten muskuloskelettalen Beschwerden“ Leitlinienreport

\begin{tabular}{|c|c|c|c|c|c|c|c|c|}
\hline & $\begin{array}{l}\text { Berater- } \\
\text { bzw. } \\
\text { Gutachter- } \\
\text { tätigkeit }\end{array}$ & $\begin{array}{l}\text { Mitarbeit in } \\
\text { einem } \\
\text { Wissenschaftlic } \\
\text { hen Beirat } \\
\text { (advisory } \\
\text { board) }\end{array}$ & $\begin{array}{l}\text { Bezahlte } \\
\text { Vortrags- } \\
\text { /oder } \\
\text { Schulungs- } \\
\text { tätigkeit }\end{array}$ & $\begin{array}{l}\text { Bezahlte } \\
\text { Autoren- } \\
\text { /oder } \\
\text { Coautoren- } \\
\text { schaft }\end{array}$ & $\begin{array}{l}\text { Forschungs } \\
\text {-vorhaben/ } \\
\text { Durchführu } \\
\text { ng } \\
\text { klinischer } \\
\text { Studien }\end{array}$ & $\begin{array}{l}\text { Eigentümer- } \\
\text { interessen } \\
\text { (Patent, } \\
\text { Urheberrecht } \\
\text { ', } \\
\text { Aktienbesitz) }\end{array}$ & Indirekte Interessen & $\begin{array}{l}\text { Von COI betroffene } \\
\text { Themen der Leitlinie }{ }^{1} \text {, } \\
\text { (Einstufung bzgl. der } \\
\text { Relevanz), } \\
\text { Konsequenz }\end{array}$ \\
\hline Schmauder & & & & & & & $\begin{array}{l}\text { Ausschuss für Betriebssicherheit des } \\
\text { Bundesministeriums für Arbeit und Soziales }\end{array}$ & Konsequenz \\
\hline $\begin{array}{l}\text { Prof. Dr. } \\
\text { Bernhard Mann }\end{array}$ & Nein & Nein & Nein & Nein & Nein & Nein & $\begin{array}{l}\text { Mitglied: Berufsverband Deutscher } \\
\text { Soziologen/Innen e. V.. } \\
\text { Deutsche Gesellschaft für Sozialmedizin und } \\
\text { Prävention e. V. }\end{array}$ & $\begin{array}{l}\text { alle (gering), keine } \\
\text { Konsequenz }\end{array}$ \\
\hline $\begin{array}{l}\text { Prof. Dr. med. } \\
\text { Thomas A. } \\
\text { Schildhauer }\end{array}$ & $\mathrm{Ja}$ & Nein & Nein & Nein & Nein & Nein & $\begin{array}{l}\text { Mitglied: Forum MedTech Pharma } \\
\text { Gesellschaft für Orthopädie und Unfallchirurgie e. V. }\end{array}$ & $\begin{array}{l}\text { alle (moderat), } \\
\text { Stimmenthaltung }\end{array}$ \\
\hline $\begin{array}{l}\text { PD. Dr.-Ing. } \\
\text { Matthias Jäger }\end{array}$ & Nein & $\mathrm{Ja}$ & Nein & Nein & $\mathrm{Ja}$ & Nein & $\begin{array}{l}\text { Mitglied: Gesellschaft für Arbeitswissenschaft e. V. } \\
\text { Deutsche Gesellschaft für Arbeitsmedizin und } \\
\text { Umweltmedizin e. V. } \\
\text { Deutsche Gesellschaft für Biomechanik e. V. }\end{array}$ & $\begin{array}{l}\text { alle (gering), keine } \\
\text { Konsequenz }\end{array}$ \\
\hline $\begin{array}{l}\text { Dr. med. } \\
\text { Alexander von } \\
\text { Glinski }\end{array}$ & Nein & Nein & Nein & Nein & Nein & Nein & $\begin{array}{l}\text { Mitglied: Gesellschaft für Orthopädie und } \\
\text { Unfallchirurgie e. V. }\end{array}$ & $\begin{array}{l}\text { alle (gering), keine } \\
\text { Konsequenz }\end{array}$ \\
\hline \multicolumn{9}{|l|}{ BeraterInnen } \\
\hline Dr.-Ing. Sascha & Nein & $\mathrm{Ja}$ & Nein & Nein & $\mathrm{Ja}$ & Nein & Mitglied: Gesellschaft für Arbeitswissenschaft e. V. & beratende Funktion \\
\hline
\end{tabular}


S2K-Leitlinien „Einsatz von Exoskeletten im beruflichen Kontext zur Primär-, Sekundär- und Tertiärprävention von arbeitsassoziierten muskuloskelettalen Beschwerden“ Leitlinienreport

\begin{tabular}{|c|c|c|c|c|c|c|c|c|}
\hline & $\begin{array}{l}\text { Berater- } \\
\text { bzw. } \\
\text { Gutachter- } \\
\text { tätigkeit }\end{array}$ & $\begin{array}{l}\text { Mitarbeit in } \\
\text { einem } \\
\text { Wissenschaftlic } \\
\text { hen Beirat } \\
\text { (advisory } \\
\text { board) }\end{array}$ & $\begin{array}{l}\text { Bezahlte } \\
\text { Vortrags- } \\
\text { /oder } \\
\text { Schulungs- } \\
\text { tätigkeit }\end{array}$ & $\begin{array}{l}\text { Bezahlte } \\
\text { Autoren- } \\
\text { /oder } \\
\text { Coautoren- } \\
\text { schaft }\end{array}$ & $\begin{array}{l}\text { Forschungs } \\
\text {-vorhaben/ } \\
\text { Durchführu } \\
\text { ng } \\
\text { klinischer } \\
\text { Studien }\end{array}$ & $\begin{array}{l}\text { Eigentümer- } \\
\text { interessen } \\
\text { (Patent, } \\
\text { Urheberrecht } \\
\text { ', Aktienbesitz) }\end{array}$ & Indirekte Interessen & $\begin{array}{l}\text { Von COI betroffene } \\
\text { Themen der Leitlinie }{ }^{1} \text {, } \\
\text { (Einstufung bzgl. der } \\
\text { Relevanz), } \\
\text { Konsequenz }\end{array}$ \\
\hline \multicolumn{9}{|l|}{ Wischniewski } \\
\hline Peter Frener & Nein & Nein & Nein & Nein & Nein & Nein & Nein & beratende Funktion \\
\hline Dr. Kai Heinrich & Nein & Nein & Nein & Nein & Nein & Nein & Nein & beratende Funktion \\
\hline $\begin{array}{l}\text { Dr.-Ing. } \\
\text { Gabriele Winter }\end{array}$ & Nein & $\mathrm{Ja}$ & Nein & $\mathrm{Ja}$ & $\mathrm{Ja}$ & Nein & $\begin{array}{l}\text { Mitglied: Gesellschaft für Arbeitswissenschaft e. V. } \\
\text { Verein Deutscher Ingenieure e. V. }\end{array}$ & beratende Funktion \\
\hline Ralf Schick & $\mathrm{Ja}$ & Nein & $\mathrm{Ja}$ & Ja & Nein & Nein & - & beratende Funktion \\
\hline $\begin{array}{l}\text { Gerhard } \\
\text { Schnalke }\end{array}$ & Nein & Nein & $\mathrm{Ja}$ & Nein & $\mathrm{Ja}$ & Nein & $\begin{array}{l}\text { Mitglied: EFL (Evaluation funktioneller } \\
\text { Leistungsfähigkeit nach Isernhagen) Bundesverband } \\
\text { e. V. } \\
\text { Verein für Prävention und Rehabilitation }\end{array}$ & beratende Funktion \\
\hline
\end{tabular}


S2K-Leitlinien „Einsatz von Exoskeletten im beruflichen Kontext zur Primär-, Sekundär- und Tertiärprävention von arbeitsassoziierten muskuloskelettalen Beschwerden“ - Leitlinienreport

\section{Verbreitung und Implementierung}

○ Konzept zur Verbreitung und Implementierung (s. DELBI-Kriterien 27, 28)

Die Publikation dieser Leitlinie erfolgt über die Internetseite der AWMF und wissenschaftliche Fachzeitschriften (Zeitschrift: Arbeitsmedizin, Sozialmedizin, Umweltmedizin sowie der Zeitschrift für Arbeitswissenschaft).

Darüber hinaus soll die Leitlinie über die beteiligten Fachgesellschaften und beratenden Institutionen, die an dieser Leitlinie mitgewirkt haben, in die betriebliche Praxis und an betriebsbetreuende Ärztinnen und Ärzte verbreitet werden. Außerdem wird auf den Internetseiten der Bundesanstalt für Arbeitsschutz und Arbeitsmedizin, der Deutschen Gesetzlichen Unfallversicherung, der Berufsgenossenschaft Handel und Warenlogistik, Berufsgenossenschaft Holz und Metall sowie der Berufsgenossenschaft Verkehrswirtschaft Post-Logistik Telekommunikation auf die Leitlinie verwiesen.

○ Unterstützende Materialien für die Anwendung der Leitlinie (s. DELBI-Kriterium 18) Nicht vorgesehen.

○ Diskussion möglicher organisatorischer und/oder finanzieller Barrieren gegenüber der Anwendung der Leitlinienempfehlungen (s. DELBI-Kriterien 19, 20) In der Leitlinie wird häufig darauf verwiesen, dass die betriebsbetreuende Ärztin oder der betriebsbetreuende Arzt eingebunden werden soll. Kleinere Betriebe haben eventuell kaum eine Möglichkeit, Ärztinnen oder Ärzte bzw. medizinisch geschultes Personal bei der Anwendung von Exoskeletten einzubinden.

○ Messgrößen für das Monitoring: Qualitätsziele, Qualitätsindikatoren (s. DELBI-Kriterium 21) Nicht vorgesehen.

\section{Gültigkeitsdauer und Aktualisierungsverfahren}

○ Datum der letzten inhaltlichen Überarbeitung und Status

Die letzte inhaltliche Überarbeitung der Leitlinie erfolgte im Mai 2020.

$\circ$ Aktualisierungsverfahren (s. DELBI-Kriterium 14)

Die Aktualisierung dieser Leitlinie ist nach drei Jahren vorgesehen.

Versions-Nummer:

Erstveröffentlichung:

\section{Nächste Überprüfung geplant:}

1.0

$05 / 2020$

Die AWMF erfasst und publiziert die Leitlinien der Fachgesellschaften mit größtmöglicher Sorgfalt - dennoch kann die AWMF für die Richtigkeit des Inhalts keine Verantwortung übernehmen. Insbesondere bei Dosierungsangaben sind stets die Angaben der Hersteller zu beachten!

Autorisiert für elektronische Publikation: AWMF online 